\title{
Desempeño de métodos moleculares para la identificación de subtipos poco comunes del virus de la hepatitis $\mathbf{C}$, genotipo 2
}

\author{
Pierina D’Angelo', Rossana Celeste Jaspe ${ }^{2}$, Carmen Luisa Loureiro², Cristina Gutiérrez¹, María Zulay \\ Sulbarán ${ }^{3}$, Yoneira Sulbarán², Félix Toro ${ }^{4}$, Flor Helene Pujol ${ }^{2}$ \\ 1 Laboratorio de Programas Especiales-Hepatitis y SIDA, Instituto Nacional de Higiene "Rafael Rangel", Caracas, \\ Venezuela \\ 2 Laboratorio de Virología Molecular, Instituto Venezolano de Investigaciones Científicas, Caracas, Venezuela \\ 3 Laboratorio de Virología, Postgrado en Biología Aplicada, Universidad de Oriente, Núcleo Sucre, Cumaná, \\ Venezuela \\ 4 Instituto de Inmunología, Universidad Central de Venezuela, Caracas, Venezuela
}

Introducción. El virus de la hepatitis C (HCV) presenta una gran variabilidad genética, con siete genotipos y numerosos subtipos. La determinación del tipo viral ha sido fundamental para la escogencia y la duración del tratamiento antiviral adecuado. En Venezuela, el genotipo 2 del HCV es relativamente diverso, siendo particularmente prevalente el subtipo 2j.

Objetivo. Evaluar el desempeño de las metodologías para la determinación del genotipo del HCV, particularmente para la identificación del subtipo 2j.

Materiales y métodos. Se determinaron el genotipo y el subtipo del HCV mediante la técnica de hibridación inversa LiPA (Line Probe Assay) y secuenciación de las regiones genómicas 5'NC y NS5B del virus.

Resultados. En 65 muestras analizadas, la metodología basada en la amplificación de la región 5’NC mostró mayor sensibilidad (100\%), en comparación con la técnica LiPA (91\%) y la secuenciación de la región NS5B (77\%). La determinación de genotipo, tomando como método de referencia la secuenciación de NS5B, mostró un alto grado de concordancia para la secuenciación de la región 5 'NC y la hibridación inversa LiPA, con $100 \%$ en la asignación de genotipos, comparado con $70 \%$ y $66 \%$ para los subtipos, respectivamente. La secuenciación de la región NS5B permitió identificar los subtipos 2j y 2s, los cuales no fueron detectados por las otras metodologías. No se observó un patrón característico para las muestras subtipo 2j en la hibridación inversa LiPA.

Conclusión. Aunque es la metodología con menor sensibilidad, la secuenciación de la región NS5B es una herramienta poderosa para la correcta discriminación de los distintos subtipos circulantes del HCV, lo cual reviste importancia epidemiológica.

Palabras clave: hepatitis C/genética; genotipo; patología molecular.

doi: https://doi.org/10.7705/biomedica.v38i0.3864

\section{Performance of molecular methods for identification of unusual subtypes of hepatitis $\mathrm{C}$ virus genotype 2}

Introduction: Hepatitis C virus (HCV) displays high genetic variability, with seven genotypes and numerous subtypes. The determination of the viral type has been essential for the selection and timing of antiviral treatment. In Venezuela, HCV genotype 2 is relatively diverse, being particularly prevalent subtype 2j.

Objective: To evaluate the performance of methodologies for genotyping HCV, particularly for identification of subtype $2 \mathrm{j}$.

Materials and methods: HCV genotype and subtype were determined by reverse hybridization technique (LiPA) and sequencing of the HCV 5'UTR and NS5B regions.

Results: A total of 65 samples from HCV-infected patients were analyzed. PCR amplifications of the 5'UTR region exhibited the highest sensitivity (100\% vs $91 \%$ for LiPA and $77 \%$ for NS5B). Genotype determination, taking as reference test NS5B, showed $100 \%$ concordance with the other methods, and $67 \%$ and $59 \%$ for subtypes with $5{ }^{\prime} \mathrm{NC}$ and LiPA, respectively. NS5B sequencing allowed the identification of subtypes $2 \mathrm{j}$ and $2 \mathrm{~s}$, which were not detected by the other methods. A specific LiPA pattern was not observed for HCV subtype 2j.

\section{Contribución de los autores:}

Félix Toro y Flor Helene Pujol: diseño del estudio

Pierina D’Angelo, Rossana Celeste Jaspe, Carmen Luisa Loureiro, Cristina Gutiérrez, María Zulay Sulbarán, Yoneira Sulbarán: recolección de datos

Todos los autores participaron en el análisis de datos y en la redacción del artículo. 
Conclusion: Although being the methodology with lowest sensitivity for amplification of HCV RNA, sequencing NS5B region remains a powerful tool for correct discrimination of the different HCV subtypes, which is of epidemiological relevance.

Key words: Hepatitis C/genetics; genotype; pathology, molecular. doi: https://doi.org/10.7705/biomedica.v38i0.3864

Existen de 130 a 150 millones de personas crónicamente infectadas con el virus de la hepatitis $C$ (HCV) (1). Esta infección crónica puede conllevar cirrosis, cáncer hepático y muerte (2-4). El HCV muestra una gran variabilidad genética, con siete genotipos y numerosos subtipos con una divergencia que alcanza el $30 \%$ en genotipos y alrededor del $15 \%$ en los subtipos $(5,6)$. Los genotipos y subtipos del HCV difieren en su distribución geográfica (7).

En Latinoamérica, la prevalencia de la infección por hepatitis $C$ oscila entre 0,3 y $0,9 \%$, con predominio de la circulación de los genotipos 1 (6-81\%), 2 $(0-33 \%)$ y $3(0-30 \%)(7-9)$. Cabe destacar que la prevalencia más alta para el genotipo 2 en la región, se encuentra en Venezuela $(8,10)$. La diversidad genética del HCV en Latinoamérica y el Caribe es más restringida que la que se observa en el continente africano, en general, con una menor variedad de subtipos y menor diversidad entre los aislamientos circulantes (11).

En Venezuela, los subtipos predominantes del HCV son el 1a, el 1b y el 2j; el genotipo 2j es el más frecuente, aun considerando el número relativamente grande de subtipos circulantes del genotipo 2. En países vecinos, como Colombia y Brasil, el subtipo 2j no se ha identificado y se reporta la circulación de subtipos más comunes, como el 2a, el $2 b$ y el $2 c(10,11)$.

Un factor ampliamente reconocido implicado en la patogénesis viral es el genotipo del HCV involucrado en la infección, según el cual la reacción al tratamiento puede variar (12). El tratamiento de la infección crónica por el HCV se ha revolucionado con el surgimiento de los antivirales de acción directa, en un intento por optimizar un tratamiento efectivo para todas las variantes del virus (13).

\footnotetext{
Correspondencia:

Flor H. Pujol, Laboratorio de Virología Molecular, Instituto Venezolano de Investigaciones Científicas, Caracas 1020-A, Venezuela

Teléfono: (58-212) 5041623; fax (58-212) 5041623

fhpujol@gmail.com

Recibido: 29/03/17; aceptado: 12/09/17
}

Como sucede con el tratamiento con el interferón 'pegilado' y la ribavirina, la selección de un antiviral de acción directa, la duración del tratamiento y la reacción virológica sostenida dependen del genotipo y el subtipo del HCV $(14,15)$. Poco se conoce sobre la distribución de más de 67 subgenotipos del HCV. Potencialmente, la diversificación del virus podría afectar las tasas de reacción virológica sostenida si surgen sustituciones asociadas con la resistencia. Estas mutaciones dependen del subtipo y afectan la efectividad del tratamiento (16).

Dado el impacto mundial de la infección por el HCV y debido a que los estudios sobre la diversidad de sus subtipos son escasos en Latinoamérica, es importante contar con herramientas para determinar el genotipo, que permitan la búsqueda de información más precisa y correcta sobre la dinámica de la distribución de los genotipos y subtipos del virus.

En este sentido, el objetivo de este estudio fue evaluar el desempeño de distintos métodos, la secuenciación de ácidos nucleicos y la hibridación inversa (17), para determinar el genotipo del $\mathrm{HCV}$ e identificar el subtipo $2 \mathrm{j}$, muy prevalente en Venezuela.

\section{Materiales y métodos \\ Población de estudio}

Previa autorización y consentimiento informado por parte de los participantes, se analizaron 65 muestras de suero o plasma provenientes de pacientes infectados con el HCV que acudieron al servicio diagnóstico entre los años 2009 y 2012. La mayoría de los pacientes eran crónicos, con diagnóstico previo de infección por el HCV y con seguimiento médico especializado para ser sometidos a tratamiento antiviral. Las muestras se procesaron inmediatamente o se mantuvieron a una temperatura de $-70{ }^{\circ} \mathrm{C}$ hasta su posterior análisis.

\section{Determinación del genotipo viral}

Las muestras se evaluaron mediante el ensayo de hibridación inversa Versant HCV Genotype 2.0 Assay (LiPA) ${ }^{\mathrm{TM}}$ (Innogenetics, distribuido por Siemens, Healthcare Diagnostic, USA). Asimismo, se evaluaron el genotipo y el subtipo mediante la secuenciación de ácidos nucleicos a partir de las 
regiones genómicas 5'NC y NS5B del HCV (10). Las secuencias de nucleótidos obtenidas fueron alineadas con un panel de secuencias de referencia depositadas en la base de datos de GenBank.

Se hizo el análisis filogenético mediante el método neighbor-joining, basado en matrices de distancias estimadas por Kimura de dos parámetros, utilizando el programa DNAman, versión 5.2.2 (Lynnon Biosoft, Canadá). Los análisis fueron sustentados con valores de confianza (bootstrap) de 1.000 réplicas y se crearon árboles filogenéticos basados estrictamente en el porcentaje de divergencia genética.

\section{Análisis estadístico}

El grado de concordancia entre las metodologías empleadas se estableció mediante el cálculo del coeficiente kappa (к) (18).

\section{Resultados}

En este estudio, se analizaron 65 muestras provenientes de sueros de pacientes que presentaron anticuerpos contra el HCV (anti-HCV). A partir de una reacción en cadena de la polimerasa (PCR) anidada en la región $5^{\prime} \mathrm{NC}$ del HCV ( $\left.5^{\prime} \mathrm{NC}\right)$, se logró identificar el genotipo circulante en el $100 \%$ de las muestras, a diferencia del $91 \%(59 / 65)$ con el ensayo de hibridación inversa (LiPA) y el $77 \%$ (50/65) al emplear la región NS5B del HCV (NS5B).

El análisis de los resultados reveló la circulación predominante de dos genotipos representados por tres subtipos, específicamente $1 \mathrm{a}, 1 \mathrm{~b}$ y $2 \mathrm{j}$. Sin embargo, la asignación del subtipo $2 \mathrm{j}$ se determinó únicamente a partir de la amplificación de la región NS5B, mientras que, con los métodos 5'NC y LiPA, los aislamientos del subtipo $2 \mathrm{j}$ fueron clasificados en su mayoría como subtipo $2 \mathrm{ac}$ o, en su defecto, como genotipo 2. Los métodos mostraron una concordancia del $100 \%$ en cuanto a la asignación de genotipos, a diferencia de lo observado en la asignación de los subtipos circulantes. Al comparar la asignación de subtipos de los métodos $5{ }^{\prime} \mathrm{NC}$ y LiPA con NS5B, se observó una concordancia de 70 y $66 \%$, respectivamente. En el cuadro 1 se muestra la correlación de las asignaciones de subtipos entre los métodos LiPA y NS5B. La discrepancia entre subtipos del HCV se detectó, particularmente, para aquellos subtipos poco comunes del genotipo 2 .

En la figura 1 se muestra el árbol filogenético usando las secuencias de la región NS5B. Se observó una gran diversidad de subtipos dentro del genotipo 2, con cinco subtipos ( $2 \mathrm{a}, 2 \mathrm{~b}, 2 \mathrm{c}, 2 \mathrm{j}$ y $2 \mathrm{~s}$ ), algunos de los cuales no fueron identificados por los otros métodos de determinación de genotipo. En cuanto a los genotipos 4, el análisis en la región NS5B permitió clasificarlos como subtipo 4d.

Debido a la gran frecuencia del subtipo $2 \mathrm{j}$ en el grupo estudiado, locual es poco común fuera de Venezuela, se quiso evaluar si existía un patrón distintivo de hibridación para este subtipo mediante el LiPA. Sin embargo, no se observó un patrón sugestivo de la presencia del genotipo $2 \mathrm{j}$, ya que los aislamientos $2 \mathrm{j}$ presentaron cuatro patrones distintos de hibridación, no pudiéndose discriminar las muestras con genotipo $2 \mathrm{j}$ de las otras con genotipo 2. Ninguna de las muestras con genotipo 2j mostró banda de hibridación en la región de la cápside. De la misma manera, las dos muestras clasificadas como genotipo 2 s por la región NS5B, mostraron el mismo patrón de bandas en la tira de nitrocelulosa con el método LiPA, siendo asignadas como genotipo 2 (figura 1).

Cuadro 1. Concordancia entre los métodos NS5B y LiPA

\begin{tabular}{|c|c|c|c|c|c|c|c|c|c|c|}
\hline \multirow{2}{*}{$\begin{array}{l}\text { Genotipo } \\
\text { Región NS5B }\end{array}$} & \multicolumn{10}{|c|}{ LiPA } \\
\hline & $1 a$ & $1 b$ & 2 & $2 a / c$ & $2 b$ & 3 & $3 a$ & 4 & $4 a / c / d$ & Total \\
\hline $1 a$ & 14 & 1 & & & & & & & & 15 \\
\hline $1 b$ & & 10 & & & & & & & & 10 \\
\hline $2 a$ & & & & 1 & & & & & & 1 \\
\hline $2 b$ & & & & & 1 & & & & & 1 \\
\hline $2 c$ & & & 3 & 3 & & & & & & 6 \\
\hline $2 \mathrm{j}$ & & & 1 & 8 & & & & & & 9 \\
\hline $2 s$ & & & 2 & & & & & & & 2 \\
\hline $3 a$ & & & & & & 1 & 3 & & & 4 \\
\hline $4 d$ & & & & & & & & 1 & 1 & 2 \\
\hline Total & 14 & 11 & 6 & 12 & 1 & 1 & 3 & 1 & 1 & 50 \\
\hline Kappa & & & & & & & & & & 0,66 \\
\hline
\end{tabular}

Las letras en negrilla corresponden a una concordancia total y, las letras en cursiva, a discrepancias en subtipo. 


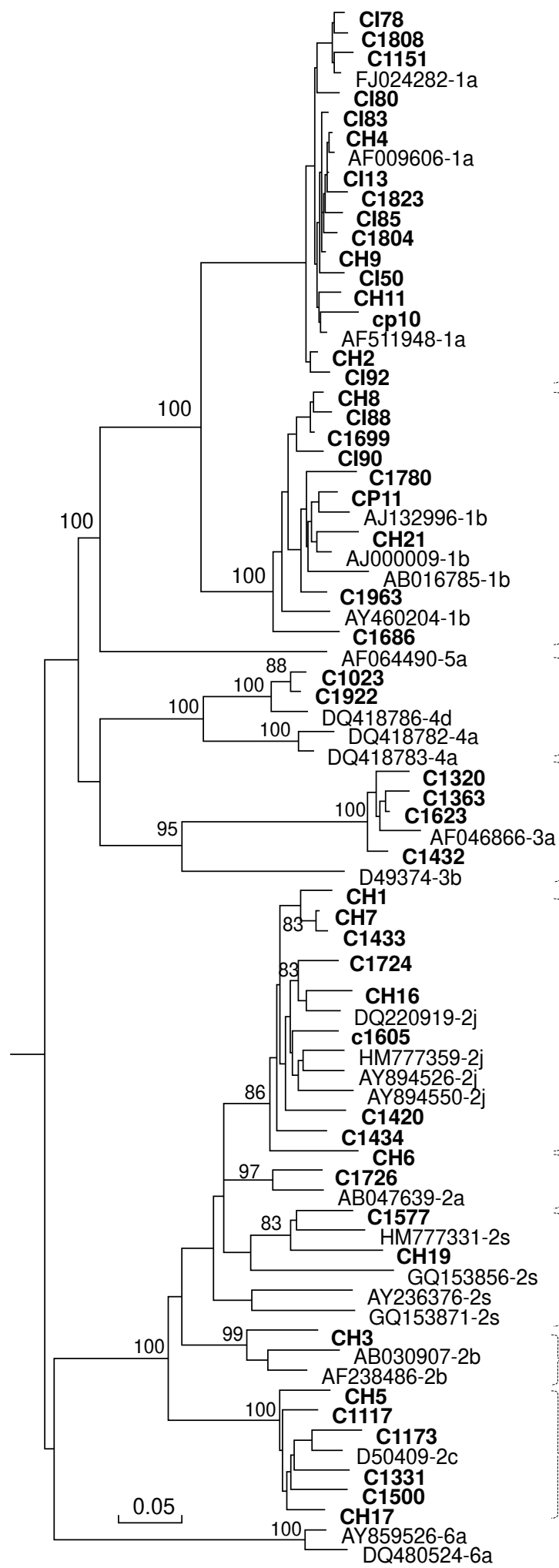

$1 \mathrm{a}$

$1 b$

G4

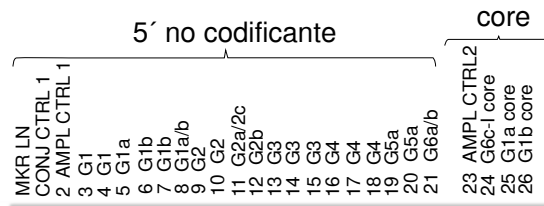

$2 a$
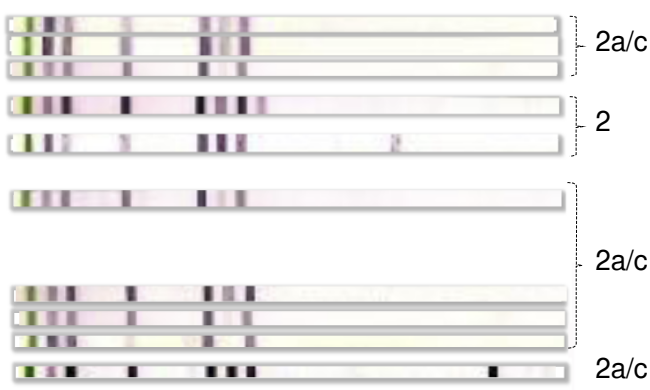

2s
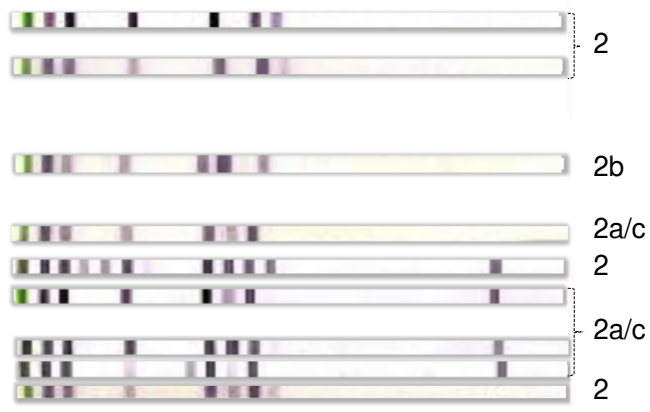

2c

2

Figura 1. Árbol filogenético de la región NS5B de aislamientos del HCV. Se estimó la distancia genética por el método de Kimura de dos parámetros y se construyó el árbol filogenético con el método de neighbor-joining. Se muestran los valores de bootstrap por encima de $80 \%$. Los aislamientos están designados con su número de acceso en el GenBank y su genotipo, salvo los aislamientos venezolanos analizados en este estudio, que se muestran en negrilla. Se muestra el patrón de bandas obtenidas por LiPA para las muestras de genotipo 2 . 


\section{Discusión}

El presente estudio permitió hacer un análisis comparativo de diferentes metodologías para determinar el genotipo circulante del HCV. La amplificación del ARN viral por los métodos LiPA y 5'NC mostró la mayor sensibilidad, de $91 \%$ y $100 \%$, respectivamente, comparada con una de $77 \%$ obtenida por el método NS5B. Estos resultados concuerdan con los reportados en otros estudios, en los cuales los métodos basados en la amplificación de la región $5^{\prime} \mathrm{NC}$ mostraron un alto grado de sensibilidad (17,19-21). No se puede descartar que el almacenamiento de las muestras o una baja carga viral hayan afectado el éxito de amplificación por medio de alguna de estas técnicas. Sin embargo, la baja sensibilidad del método NS5B ha sido reportada previamente, en particular, para aislamientos virales del genotipo 2 (10).

En términos de calidad de asignación de genotipos, los resultados mostraron el $100 \%$ de correlación por los diferentes métodos empleados. Este alto grado de correlación es similar a lo reportado por otros autores $(17,22)$. La mayoría de las discrepancias observadas en los subtipos con las determinaciones por LiPA y $5^{\prime} \mathrm{NC}$ respecto al método de referencia, se presentaron en aislamientos del genotipo 2, lo que concuerda con los reportes de Cai, et al., en 2013, en los cuales la identificación de los subtipos con la técnica LiPA y la secuenciación de la región NS5B alcanzó una concordancia de solo $45 \%$, en el caso del genotipo 2 (23). Welzel, et al., en 2017, observaron una mayor discordancia en la genotipificación de aislamientos del genotipo 2, al comparar el método LiPA con la secuenciación, probablemente debido a la gran diversidad de subtipos dentro de este genotipo (16).

El análisis filogenético de la región NS5B permitió identificar una gran diversidad de subtipos presentes en los aislamientos venezolanos del genotipo 2; además de la asignación de subtipos comunes, como $2 \mathrm{a}, 2 \mathrm{~b}$ y $2 \mathrm{c}$, se identificó la circulación de los subtipos 2j y 2s. La bondad de la técnica de NS5B para identificar subtipos poco comunes ha sido descrita por varios autores; por ejemplo, Margall, et al., detectaron los subtipos $2 \mathrm{j}$ y $2 \mathrm{q}$ en muestras circulantes en España (24). En Venezuela, la alta prevalencia del subtipo 2j permitió la secuenciación del genoma completo de este subtipo para su identificación (10). En Europa, se ha encontrado el genotipo 2j en España y Francia, con prevalencias que oscilan entre 0,9 y $2,3 \%$. (24). En Argentina, se identificó un aislamiento de subtipo $2 \mathrm{j}$ en una población de donantes voluntarios (25). El subtipo $2 s$, aunque no es muy frecuente en Venezuela, ha mostrado ser recurrente en distintas regiones del país $(10,26)$. Las secuencias venezolanas del $\mathrm{HCV}$ de genotipo 2j se agruparon dentro de un clado monofilético (10); a pesar de ello, se observaron diversos patrones de hibridación mediante la técnica LiPA.

La relativa alta prevalencia del genotipo 2 en Venezuela, con respecto a otros países suramericanos, podría deberse a varios factores. En primer lugar, podría deberse a la relativa baja frecuencia de subtipos que circulan entre usuarios de drogas intravenosas, como el subtipo $3 a$, ya que la frecuencia de su uso es relativamente menor en Venezuela (27). En segundo lugar, no se puede descartar una ventaja en la diseminación del subtipo 2j en Venezuela por haber sido introducido en la época colonial, mientras que los otros subtipos, como 1a y 1b, fueron introducidos el siglo pasado (10). Finalmente, se desconoce si existe un factor de riesgo asociado con la infección por el subtipo 2j; no se descarta que la transmisión sexual pueda influir (10), lo cual podría estar afectando la prevalencia de este subtipo.

El conocer el subtipo del HCV para el manejo clínico de los pacientes está cobrando importancia con el advenimiento de fármacos antivirales de acción directa ya que, por ejemplo, los pacientes infectados con el subtipo 1a tienden a mejorar de forma más lenta que los infectados con el subtipo 1b $(14,28)$. Se desconoce si esta variación podría asociarse con una mayor resistencia a los nuevos tratamientos (29). La asignación de genotipos y subtipos del virus es también importante para diversos propósitos de investigación, incluyendo estudios filogenéticos, epidemiológicos y de la historia natural de la infección.

Por otra parte, se desconoce la diversidad de subtipos del HCV en muchos países latinoamericanos. Así como ocurre en Venezuela, en otros países de la región podrían presentarse subtipos poco frecuentes del HCV. Es por ello que se recomienda que en cada país se evalúe la posibilidad de estudiar la secuenciación de las variantes circulantes, bien sea con fines epidemiológicos o de rutina clínica, usando, por ejemplo, la secuenciación en la región NS5B, para tener una visión de la variedad de subtipos que circulan, ya que esto podría tener implicaciones de adecuación del tratamiento antiviral.

\section{Conflicto de intereses}

Los autores declaran no tener conflicto de intereses. 


\section{Financiación}

Este proyecto fue parcialmente financiado por el proyecto Misión Ciencia: Tecnología para el diagnóstico molecular de enfermedades transmisibles, FONACIT, Venezuela.

\section{Referencias}

1. Lanini S, Easterbrook PJ, Zumla A, Ippolito G. Hepatitis C: Global epidemiology and strategies for control. Clin Microbiol Infect. 2016;22:833-8. https://doi.org/10.1016/j. cmi.2016.07.035

2. Simmonds P. Genetic diversity and evolution of hepatitis $C$ virus - 15 years on. J Gen Virol. 2004;85:3173-88. https:// doi.org/10.1099/vir.0.80401-0

3. Hnatyszyn HJ. Chronic hepatitis $C$ and genotyping: The clinical significance of determining HCV genotypes. Antivir Ther. 2005;10:1-11.

4. Tellinghuisen TL, Evans MJ, von Hahn T, You S, Rice CM. Studying hepatitis $C$ virus: Making the best of a bad virus. J Virol. 2007;81:8853-67. https://doi.org/10.1128/JVI. 00753-07

5. Smith DB, Bukh J, Kuiken C, Muerhoff AS, Rice CM, Stapleton JT. Expanded classification of hepatitis $C$ virus into 7 genotypes and 67 subtypes: Updated criteria and genotype assignment web resource. Hepatology. 2014;59: 318-27. https://doi.org/10.1002/hep.26744

6. Timm J, Roggendorf M. Sequence diversity of hepatitis C virus: Implications for immune control and therapy. World J Gastroenterol. 2007;13:4808-17. https://doi.org/10.3748/ wjg.v13.i36.4808

7. Gower E, Estes C, Blach S, Razavi-Shearer K, Razavi H. Global epidemiology and genotype distribution of the hepatitis C virus infection. J Hepatol. 2014;61:S45-57. https://doi.org/10.1016/j.jhep.2014.07.027

8. Polaris Observatory HCV Collaborators. Global prevalence and genotype distribution of hepatitis $C$ virus infection in 2015: A modelling study. Lancet Gastroenterol Hepatol. 2017;2:161-76. https://doi.org/10.1016/S24681253(16)30181-30189

9. Alvarado-Mora MV, Pinho JR. Epidemiological update of hepatitis B, C and Delta in Latin America. Antivir Ther. 2013; 18:429-33. https://doi.org/10.3851/IMP2595

10. Sulbarán MZ, Di Lello FA, Sulbarán Y, Cosson C, Loureiro CL, Rangel HR, et al. Genetic history of hepatitis $C$ virus in Venezuela: High diversity and long time of evolution of HCV genotype 2. PLoS One. 2010;5:e14315. https://doi.org/10.1371/journal.pone.0014315

11. Cristina J. Genetic diversity and evolution of hepatitis C virus in the Latin American region. J Clin Virol. 2005; 34(Suppl.2):S1-7. https://doi.org/10.1016/S1386-6532(05) 00393-8

12. Cuypers L, Ceccherini-Silberstein F, van Laethem $\mathrm{K}$, Li G, Vandamme AM, Rockstroh JK. Impact of HCV genotype on treatment regimens and drug resistance: A snapshot in time. Rev Med Virol. 2016;26:408-34. https://doi. org/10.1002/rmv.1895
13. Asselah T, Boyer N, Saadoun D, Martinot-Peignoux M, Marcellin P. Direct-acting antivirals for the treatment of hepatitis $C$ virus infection: Optimizing current IFNfree treatment and future perspectives. Liver Int. 2016;36 (Suppl.1):47-57. https://doi.org/ 10.1111/liv.13027

14. Jiménez-Pérez M, González-Grande R, España Contreras P, Pinazo Martínez I, de la Cruz Lombardo J, Olmedo Martín R. Treatment of chronic hepatitis $\mathrm{C}$ with direct-acting antivirals: The role of resistance. World $\mathrm{J}$ Gastroenterol. 2016;22:6573-81. https://doi.org/10.3748/wjg.v22.i29.6573

15. Foster GR, Afdhal N, Roberts SK, Brau N, Gane EJ, Pianko S. Sofosbuvir and Velpatasvir for HCV genotype 2 and 3 infection. N Engl J Med. 2015;373:2608-17. https:// doi.org/10.1056/NEJMoa1512612

16. Welzel TM, Bhardwaj N, Hedskog C, Chodavarapu K, Camus G, McNally J, et al. Global epidemiology of HCV subtypes and resistance-associated substitutions evaluated by sequencing-based subtype analyses. J Hepatol. 2017;67: 224-36. https://doi.org/10.1016/j.jhep.2017.03.014

17. Verbeeck J, Stanley MJ, Shieh J, Celis L, Huyck E, Wollants E, et al. Evaluation of Versant hepatitis $C$ virus genotype assay (LiPA) 2.0. J Clin Microbiol. 2008;46:19016. https://doi.org/10.1128/JCM.02390-07

18. Viera AJ, Garrett JM. Understanding interobserver agreement: The kappa statistic. Fam Med. 2005;37:360-3.

19. Laperche S, Lunel F, Izopet J, Alain S, Dény P, Duverlie G, et al. Comparison of hepatitis C virus NS5B and 5' noncoding gene sequencing methods in a multicenter study. J Clin Microbiol. 2005;43:733-9. https://doi.org/10.1128/JCM. 43.2.733-739.2005

20. Scott JD, Gretch DR. Molecular diagnostics of hepatitis C virus infection: A systematic review. JAMA. 2007;297:72432. https://doi.org/10.1001/jama.297.7.724

21. Baclig MO, Chan VF, Ramos JD, Gopez-Cervantes J, Natividad FF. Correlation of the 5'untranslated region (5'UTR) and non-structural 5B (NS5B) nucleotide sequences in hepatitis $\mathrm{C}$ virus subtyping. Int $\mathrm{J}$ Mol Epidemiol Genet. 2010;1:236-44.

22. Martró E, González V, Buckton AJ, Saludes V, Fernández G, Matas L, et al. Evaluation of a new assay in comparison with reverse hybridization and sequencing methods for hepatitis $\mathrm{C}$ virus genotyping targeting both 5' noncoding and nonstructural $5 \mathrm{~b}$ genomic regions. J Clin Microbiol. 2008;46:192-7. https://doi.org/10.1128/JCM.01623-07

23. Cai Q, Zhao Z, Liu Y, Shao X, Gao Z. Comparison of three different HCV genotyping methods: Core, NS5B sequence analysis and line probe assay. Int J Mol Med. 2013;31:34752. https://doi.org/10.3892/ijmm.2012.1209

24. Margall N, March F, Español M, Torras X, Gallego A, Coll P. Two unusual hepatitis $C$ virus subtypes, $2 \mathrm{j}$ and $2 \mathrm{q}$, in Spain: Identification by nested-PCR and sequencing of a NS5B region. J Virol Methods. 2015;223:105-8. https://doi. org/10.1016/j.jviromet.2015.07.022

25. del Pino N, Oubiña JR, Rodríguez-Frías F, Esteban JI, Buti M, Otero T, et al. Molecular epidemiology and putative origin of hepatitis $\mathrm{C}$ virus in random volunteers from Argentina. World J Gastroenterol. 2013;19:5813-27. https:// doi.org/10.3748/wjg.v19.i35.5813 
26. Sulbarán MZ, Montaño L, Sulbarán Y, Loureiro CL, Flores CR, Farías Y, et al. Caracterización molecular del virus de la hepatitis $C$ en pacientes remitidos al Laboratorio Regional de Salud Pública del Hospital Universitario "Antonio Patricio de Alcalá", Cumaná, Venezuela. Invest Clin. 2016:57:13-24

27. Jaspe RC, Sulbarán YF, Loureiro CL, Martínez N, Devesa M, Rodríguez $\mathbf{Y}$ et al. Genetic diversity of hepatitis B virus and hepatitis $C$ virus in human immunodeficiency virus type 1-co-infected patients from Venezuela. J Med Microbiol. 2014:63:1099-104. https://doi.org/10.1099/jmm.0.067496-0
28. Chevaliez S, Bouvier-Alias M, Brillet R, Pawlotsky JM. Hepatitis $C$ virus (HCV) genotype 1 subtype identification in new HCV drug development and future clinical practice. PLoS One. 2009;4:1-9. https://doi.org/10.1371/journal. pone.0008209

29. Le Guillou-Guillemette H, Vallet S, Gaudy-Graffin C, Payan C, Pivert A, Goudeau A, et al. Genetic diversity of the hepatitis $C$ virus: Impact and issues in the antiviral therapy. World J Gastroenterol. 2007;13:2426. https://doi. org/10.3748/wjg.v13.i17.2416 